\title{
Stimulating brain recovery after stroke using theranostic albumin nanocarriers loaded with nerve growth factor in combination therapy
}

Tivadar Feczkó ${ }^{1,2,3}$, Albrecht Piiper ${ }^{1}$, Saema Ansar ${ }^{4}$, Frank W Blixt ${ }^{4}$, Mukul Ashtikar ${ }^{5}$, Susanne Schiffmann ${ }^{5}$, Thomas Ulshöfer ${ }^{5}$, Michael J. Parnham ${ }^{5}$, Yifat Harel ${ }^{6}$, Liron Limor Israel ${ }^{6}$, Jean-Paul Lellouche ${ }^{6}$, Matthias G. Wacker ${ }^{5,7^{*}}$

1: Department of Medicine 1, University Hospital Frankfurt, Frankfurt, Germany

2: Institute of Materials and Environmental Chemistry, Research Centre for Natural Sciences, Hungarian Academy of Sciences, Budapest, Hungary

3: Research Institute of Biomolecular and Chemical Engineering, University of Pannonia, Veszprém, Hungary

4: Department of Clinical Sciences, Lund University, Lund, Sweden

5: $\quad$ Fraunhofer Institute for Molecular Biology and Applied Ecology, Translational Medicine and Pharmacology, Frankfurt/Main

6: Department of Chemistry, Bar llan University, Israel

7: Institute of Pharmaceutical Technology, Goethe University, Frankfurt/Main

${ }^{*}$ Corresponding author

\section{Dr. Matthias G. Wacker}

Pharmaceutical Technology and Nanosciences

Fraunhofer-Institute for Molecular Biology and Applied Ecology (IME)

Translational Medicine and Pharmacology (TMP)

Building N230, Room 2.03

Max-von-Laue-Straße 9, D-60438 Frankfurt/Main

Phone +4969798-29691

Fax $\quad+4969798-29694$

www.ime.fraunhofer.de 


\section{First revision}

\section{Abstract}

For many years, delivering drug molecules across the blood brain barrier has been a major challenge. The neuropeptide nerve growth factor is involved in the regulation of growth and differentiation of cholinergic neurons and holds great potential in the treatment of stroke. However, as with many other compounds, the biomolecule is not able to enter the central nervous system. In the present study, nerve growth factor and ultra-small particles of iron oxide were co-encapsulated into a chemically crosslinked albumin nanocarrier matrix which was modified on the surface with apolipoprotein $\mathrm{E}$. These biodegradable nanoparticles with a size of $212 \pm 1 \mathrm{~nm}$ exhibited monodisperse size distribution and low toxicity. They delivered NGF through an artificial blood brain barrier and were able to induce neurite outgrowth in PC12 cells in vitro. In an animal model of stroke, the infarct size was significantly reduced compared to the vehicle control. The combination therapy of NGF and the small-molecular MEK inhibitor U0126 showed a slight but not significant difference compared to U0126 alone. However, further in vivo evidence suggests the hypothesis that successful delivery of the neuropeptide is possible as well as the synergism between those two treatments.

\section{Keywords:}

BBB, brain, nanoparticles, drug delivery, theranostic, NGF, MEK 


\section{First revision}

\section{Abbreviations}

ApoE3

BBB

CAN

DCM

DMSO

EDC

ERK

HSA

MALHEX-NH-PEG-NHS

MEK

mPEG-SPA-5000

MRI

NGF

NHS

PAR

PBCA

PBS

PDI

PEG

PVA

SEM

TEM

USPIO
Apolipoprotein E3

blood brain barrier

cerium ammonium nitrate

dichloromethane

dimethyl sulfoxide

1-ethyl-3(3-dimethylaminopropyl) carbodiimide

Extracellular signal-regulated kinase

human serum albumin

Maleinimidohexanoic- $\omega-N H S$ PEG

mitogen-activated protein kinase kinase

Succinimidyl propionic acid PEG NHS

magnetic resonance imaging

nerve growth factor

$\mathrm{N}$ - hydroxysuccinimide

passive avoidance reflex

poly(butyl cyanoacrylate)

phosphate buffered saline

polydispersity index

polyethylene glycol

polyvinyl alcohol

scanning electron microscope

transmission electron microscopy

ultra-small superparamagnetic iron oxide 


\section{Introduction}

As one of the most important neuropeptides, nerve growth factor (NGF) is involved in the regulation of growth and differentiation of cholinergic neurons [1]. Further, it plays a major role in cell survival after ischemic stroke which is a leading cause of death globally [2]. A significant reduction of delayed neuronal death was observed after the administration of NGF into the cerebral ventricle of rats [3] and even some early clinical evidence highlights a beneficial effect on recovery of hypoxic-ischemic brain injuries and cerebral perfusion $[4,5]$.

Despite the great potential in the treatment of stroke, NGF is not able to pass the bloodbrain barrier (BBB) which makes clinical administration dependent on invasive neurosurgical procedures $[6,7]$. The BBB is created by the cerebral endothelial cells forming tight junctions and sealing the paracellular pathway into the brain [8]. As a consequence, the composition of the extracellular fluid within the central nervous system (CNS) can be precisely controlled [8]. The endothelial tissue exhibits polarized expression of transport proteins [8]. Some of these transporters were addressed using nanocarrier systems modified on their surface with targeting moieties such as transferrin [9], insulin [10] or the apolipoproteins (Apo) E and A-1 [11, 12]. Poly(butyl cyanoacrylate) (PBCA) nanoparticles coated with polysorbate 80 effectively delivered NGF to the CNS in an animal model of Alzheimer's disease [13].

This coating leads to an adsorption of Apo A-1, B, and E [14]. Kreuter and co-workers confirmed an active endocytotic uptake mechanism of ApoE-conjugated albumin nanoparticles in vitro [15] and in vivo [12]. In this context, the low density lipoprotein receptor (LRP1) was held responsible for the uptake [15]. Human serum albumin (HSA) is the most abundant blood protein and has been used as a carrier material for a number of therapeutic agents such as survivin miRNA plasmid [16], polo-like kinase [17], doxorubicin [18, 19] or paclitaxel [20].

Exploring the biodistribution of nanocarriers is an important requirement for clinical translation of nanocarrier delivery. In this context, ultra-small superparamagnetic particles of iron oxide (USPIO) were encapsulated into HSA nanoparticles serving as contrast agents for magnetic resonance (MR) imaging [21, 22]. A novel species of 
maghemite core particles was used [23-25]. The resulting diagnostic HSA nanocarriers were successfully tested in vivo [21].

In the present study, advanced theranostic nanocarriers for the treatment of stroke were synthesized by co-desolvation of NGF and HSA in the presence of cerium ammonium nitrate (CAN)-stabilized USPIO. The surface of resulting nanocarriers was functionalized using apolipoprotein $\mathrm{E}$ to facilitate an active transport into the brain. After a detailed physicochemical characterization, the nanocomposites were evaluated in vitro and in vivo. Biocompatibility of the nanocarriers as well as the bioactivity of NGF were confirmed in rat pheochromocytoma (PC12) cells. The nanoparticles effectively induced neurite outgrowth without exhibiting significant cytotoxicity. Drug release was investigated using the dispersion releaser technology [26, 27] The transport of NGF into the brain was confirmed in vitro in bEnd3 cells. Finally, a combination of the nanocarriers with the small-molecular mitogen-activated protein kinase (MEK) inhibitor U0126 was tested in an in vivo model of stroke. U0126 protects the brain against ischemic brain damage and a synergistic effect was assumed [28, 29],

\section{Materials and methods}

\subsection{Materials}

HSA, glutaraldehyde $25 \%$ aqueous solution, D-trehalose, saccharose, and mannitol were obtained from Sigma Aldrich (Munich, Germany). Nerve growth factor was supplied by Alomone Labs (Jerusalem, Israel). Apolipoprotein E3 was purchased from Peprotech (Hamburg, Germany). ATTO647N was purchased from ATTO-TEC GmbH (Siegen, Germany). 2-Iminothiolane HCL (Traut' s reagent) was obtained from Thermo Scientific (USA). Succinimidyl propionic acid PEG NHS (mPEG-SPA-5000) was given by NANOCS (USA). a-Maleinimidohexanoic-w-NHS PEG (MALHEX-NH-PEG-NHS) was manufactured by RAPP Polymere (Tübingen, Germany). Trypsinogen and proteinase $\mathrm{K}$ for the release experiments was purchased from Sigma Aldrich (Darmstadt, Germany). 


\subsection{Synthesis of $\mathrm{Ce} 3 / 4+-$ cation-doped maghemite iron oxide particles}

The USPIO were synthesized using a nanofabrication method described previously [23]. In brief, $\mathrm{FeCl}_{3} \cdot 6 \mathrm{H}_{2} \mathrm{O}$ ( $\mathrm{Fe}^{3+}$ cations, $240.0 \mathrm{mg}, 0.9 \mathrm{mmol}$ ) salt was dissolved in deoxygenated MilliQ water $(4.5 \mathrm{~mL})$ and mixed with a similar aqueous solution of $\mathrm{FeCl}_{2} \bullet 4 \mathrm{H}_{2} \mathrm{O}\left(\mathrm{Fe}^{2+}\right.$ cations, $\left.97.5 \mathrm{mg}, 0.45 \mathrm{mmol}, 4.5 \mathrm{~mL} \mathrm{H} 2 \mathrm{O}\right)$. This hybrid iron salt aqueous medium ( $\mathrm{N}_{2}$ atmosphere) was first ultrasonicated (Bransonic ${ }^{\circledR}$ ultrasonic cleaner bath, 2510E MTH model, $42 \mathrm{KHz}$ at full power) over a time period of 5 to $10 \mathrm{~min}$ at room temperature before introducing $24 \%$ of an aqueous $\mathrm{NH}_{4} \mathrm{OH}(0.75 \mathrm{~mL})$ phase (one shot injection) causing an immediate black precipitation of corresponding magnetite $\left(\mathrm{Fe}_{3} \mathrm{O}_{4}\right)$ nanoparticles. The procedure was followed by 19 additional minutes of ultra-sonication. Thus resulting $\mathrm{Fe}_{3} \mathrm{O}_{4}$ nanoparticles were transferred into a glass bottle $(100 \mathrm{~mL}$ ), magnetically decanted (with a strong external magnet), and washed with $\mathrm{dd}_{2} \mathrm{O}(3 \times 40 \mathrm{~mL})$ until neutral $\mathrm{pH}$ was reached. The resulting black welldispersed magnetite nanoparticles were stored $(30 \mathrm{~mL}$ nanoparticles suspension in $\mathrm{ddH}_{2} \mathrm{O}$ ) before further processing. The ageing storage process was performed for a minimum of $2 \mathrm{~h}$ (room temperature) of storage time at room temperature.

Afterwards, the former aqueous magnetite nanoparticles suspension $(30 \mathrm{~mL}, 4.22 \mathrm{~h}$ ageing time) was magnetically decanted to separate magnetite nanoparticles from the aqueous phase. Cerium-containing inorganic CAN oxidant reagent [( $\left.\left(\mathrm{NH}_{4}\right)_{2} \mathrm{Ce}(\mathrm{IV})\left(\mathrm{NO}_{3}\right)_{6}\right], 500.0 \mathrm{mg}, 0.912 \mathrm{mmol}$ was dissolved in $6.0 \mathrm{~mL}$ of acetone and introduced to the decanted magnetite nanoparticles, followed by an addition of $18 \mathrm{~mL}$ of degassed MilliQ water. Finally, the corresponding multi-phase mixture was ultrasonicated using a high-power sonicator $\left(\right.$ Sonics ${ }^{\circledR}$, Vibra cell, 750 Watt, power modulator set-up at $25 \%)$ - Titanium horn $\left(0.5 \mathrm{~h}, 0^{\circ} \mathrm{C}\right.$ - Inert argon atmosphere).

\subsection{Preparation of theranostic core particles}

To prepare the theranostic core particle, an amount of $25 \mathrm{mg}$ of HSA (fraction V, purity 96-99\%) was dissolved in $0.125 \mathrm{~mL}$ of MilliQ water and $0.25 \mathrm{ml}$ of a dialysed NGF solution ( $1 \mathrm{mg} / \mathrm{ml})$ was added. Afterwards, $10 \mu \mathrm{L}$ of a $1 \mathrm{M}$ sodium chloride solution and $101 \mu \mathrm{L}$ of a CAN- $-\mathrm{Fe}_{2} \mathrm{O}_{3}$ suspension comprising $0.5 \mathrm{mg}$ of iron were added. The $\mathrm{pH}$ was adjusted to 8.0 and the suspension was divided into 2 batches. To each batch, a total amount of $2 \mathrm{~mL}$ of ethanol $96 \%(\mathrm{v} / \mathrm{v})$ was added using a peristaltic pump (Ismatec Laboratoriumstechnik $\mathrm{GmbH}$, Wertheim, Germany) at a rate of $1.1 \mathrm{~mL} / \mathrm{min}$ while 
stirring the dispersion continuously at $400 \mathrm{rpm}$ on a magnetic stirring plate. Finally, a stoichiometric amount of glutaraldehyde corresponding to the number of amino groups in the HSA molecule was added to crosslink the protein. Again, the particle suspension was continuously stirred for 4 hours. The nanoparticles were centrifuged using an Eppendorf centrifuge 5424 R (Eppendorf AG, Hamburg, Germany) at 20,000 rpm for $25 \mathrm{~min}$, washed trice and redispersed in MilliQ water or phosphate buffered saline $(\mathrm{pH}=7.4, \mathrm{PBS})$.

\subsection{Surface modification of theranostic core particles}

The surface of theranostic HSA nanoparticles was modified as described previously $[11,12]$. For this purpose, the nanoparticles were activated using the bi-functional crosslinker MALHEX-NH-PEG-NHS. After thiolation of the ligand, ApoE was conjugated to the particle surface using the maleimide active group of the crosslinking agent.

In brief, an amount of $20 \mathrm{mg}$ of the theranostic core particles in $1.0 \mathrm{~mL}$ of PBS (pH 8.0) was mixed with $1.0 \mathrm{~mL}$ of PBS $(\mathrm{pH} 8.0)$ that contained a 10 -fold molar excess of MALHEX-NH-PEG-NHS. The suspension was agitated for 1 hour $\left(600 \mathrm{rpm}, 20^{\circ} \mathrm{C}\right)$ in a thermomixer (Eppendorf AG, Hamburg, Germany). Afterwards, the suspension was dialysed against $250 \mathrm{ml}$ MilliQ water for 2 hours and against $400.0 \mathrm{ml}$ of $0.1 \mathrm{M} \mathrm{NaHCO}_{3}$ buffer ( $\mathrm{pH}$ 8.0) for 2 hours, respectively. In both cases, a dialysis membrane with a molecular weight cut-off (MWCO) of $10 \mathrm{kDa}$ was used.

The thiolation of ApoE was achieved by incubating $1.0 \mathrm{mg}$ of the protein with a 50 -fold molar excess of Traut's reagent in PBS over a time period of 2 hours at $600 \mathrm{rpm}$ in a thermomixer (Eppendorf AG, Hamburg, Germany). Afterwards, ApoE was dialyzed against $200 \mathrm{ml}$ MilliQ water using a membrane with $3.5 \mathrm{kDa}$ MWCO for 2 hours and against $200.0 \mathrm{ml} 0.1 \mathrm{M} \mathrm{NaHCO}_{3}$ buffer ( $\mathrm{pH} 8.0$ ) for 2 hours, respectively.

Finally, the ApoE solution was incubated with the activated theranostic core particles for $18 \mathrm{~h}$ at $600 \mathrm{rpm}$ and $20{ }^{\circ} \mathrm{C}$ in a thermomixer system (Eppendorf AG, Hamburg, Germany). The resulting targeted theranostic particles were dialyzed against $500.0 \mathrm{ml}$ of MilliQ water using a membrane with a MWCO of $100 \mathrm{kDa}$ for 2 hours.

For the cell-based in vitro assays, targeted theranostic nanocarriers were labelled using the amino-reactive fluorescent dye ATTO-647N. In this context, $0.5 \mathrm{mg}$ of the nanoparticles in PBS $(0.5 \mathrm{ml}, \mathrm{pH} 8.0)$ was mixed with $0.065 \mathrm{~mL}$ of an ATTO-647N solution in DMSO $(0.1 \mathrm{mg} / \mathrm{ml})$, and agitated for $50 \mathrm{~min}$ at $600 \mathrm{rpm}$ and $20{ }^{\circ} \mathrm{C}$ in a 
thermomixer (Eppendorf AG, Hamburg, Germany). The nanoparticles were purified by repeated steps of centrifugation (25 min, 20,000 rpm, centrifuge $5424 \mathrm{R}$, Eppendorf AG, Hamburg, Germany) and redispersion in MilliQ water.

As a control, HSA nanoparticles carrying PEG chains on their surface were prepared using the mono-functional crosslinking agent mPEG-SPA-5000 to the particle surface under similar conditions.

\subsection{Characterization of theranostic particles}

The morphology of targeted theranostic nanocarriers was investigated using a FEI Morgagni 268D transmission electron microscope (FEI, Hillsboro, USA).

The particle size and size distribution were determined using the dynamic light scattering method with a Zetasizer Nano ZS (Malvern Instruments, Malvern, UK) equipped with a backscatter detector at an angle of $173^{\circ}$. The particles were characterized for the volume mean diameter and polydispersity index (PDI).

\subsection{Storage stability of theranostic particles}

To convert the targeted theranostic nanocarriers into a stable solid, form freeze-drying was applied. Mannitol, sucrose, and trehalose were evaluated as lyoprotectants at a concentration of $3 \%(\mathrm{w} / \mathrm{v})$. For this purpose, a volume of $1.0 \mathrm{~mL}$ containing $10 \mathrm{mg} / \mathrm{mL}$ of targeted theranostic particles and a lyoprotectant was freeze-dried in a Christ Epsilon 2-7 freeze dryer (Martin Christ GmbH, Osterrode, Germany) as described previously $[22,30]$. The physical stability of the targeted theranostic nanocarriers was investigated after 6 months of storage at $4{ }^{\circ} \mathrm{C}$ and reconstitution in the same volume of MilliQ water. The dynamic light scattering method was used to evaluate the size change due to the lyophilisation.

\subsection{Magnetization of theranostic nanocarriers}

The specific (mass dependant) magnetization of the freeze-dried targeted theranostic nanocarriers was determined using a superconducting quantum interference device (SQUID) magnetometer (Quantum Design Inc., San Diego, USA). The samples were fixed in a gelatine capsule and pulled through the induction coils (vibrating sample mode) of the magnetometer at constant temperature $(300 \mathrm{~K})$ and various constant external fields. 


\subsection{Determination of iron content of theranostic particles}

The iron content of the theranostic nanocarriers was quantified by inductively coupled plasma optical emission spectroscopy (ICP-OES) using a Spectro Genesis simultaneous spectrometer with axial plasma observation (Spectro Analytical Instruments GmbH, Kleve, Germany).

Multi-elemental standard solutions for ICP calibration (Merck KGaA, Darmstadt, Germany) was used for calibration. The detection limit of iron was calculated according to equation 1 :

$$
\text { Limit of detection }=\text { Background signal }+3 * S D_{\text {Background }} * f_{\text {dilution }}
$$

A known amount of theranostic nanocarriers was dissolved in $5 \mathrm{M}$ hydrochloric acid and diluted to be in the applied calibration range.

\subsection{Release from the HSA nanoparticle system using the dispersion releaser technology}

The release from HSA nanoparticles was determined using the dialysis-based dispersion releaser technology as described earlier by Janas et al. [26]. Since the testing requires relevant amounts of the protein, trypsinogen (molecular weight $24 \mathrm{kDa}$, isoelectric point 9.3) was selected as a model protein for NGF (molecular weight 26 $\mathrm{kDa}$, isoelectric point 9.3).

For this purpose, the protein was labelled using a 3-fold molar excess of ATTO-647$\mathrm{N}$-hydroxysuccinimde. Unbound fluorescent label was separated from labelled protein by using G-25 Sephadex size exclusion chromatography column (GE Healthcare Life Sciences, Buckinghamshire, UK). Subsequently diluted protein solutions obtained were concentrated by using Amicon Ultra centrifugal protein concentrators (Sigma Aldrich, Darmstadt, Germany). Desolvation and particle purification was carried as described in the section 2.3 above.

The purified particles were redispersed in $1 \mathrm{ml}$ of MilliQ water and a volume of $300 \mu \mathrm{l}$ was introduced to the donor compartment. Acceptor medium for the release study was PBS. A semipermeable $100 \mathrm{kDa}$ cellulose ester dialysis membrane was used to separate donor and acceptor compartment of the dispersion releaser. Volumes of $500 \mu \mathrm{l}$ were collected at predefined time points from the acceptor compartment. After 6 hours, a small quantity (approximately 15 units of enzyme in $50 \mu \mathrm{l}$ of volume) of 
proteinase $\mathrm{K}$ solution was added to the donor compartment to change from a diffusionbased to a degradation-based release mechanism [31].

\subsection{PC12 cytotoxicity and cellular bioactivity assay}

PC12 cells were cultured with Dulbecco's modified Eagle's medium containing $5 \%(\mathrm{v} / \mathrm{v})$ fetal bovine serum (FBS), 10\% (v/v) horse serum, and 1\% (v/v) penicillin-streptomycin $\left(37{ }^{\circ} \mathrm{C}, 5 \% \mathrm{CO}_{2}\right)$. The cytocompatibility of targeted theranostic nanocarriers was analysed using an 3-[4,5-dimethylthiazole-2-yl]-2,5-diphenyltetrazolium bromide (MTT) assay in PC12 cells. Pre-cultured cells were seeded at a density of $5 \times 10^{4}$ per well in a 96-well plate and cultured for $24 \mathrm{~h}$. An amount of 10, 25 and $50 \mu \mathrm{g}$ of targeted theranostic nanocarriers was added to each well in a volume of $5 \mu$ of PBS. The cells were incubated for 24,48 , and $72 \mathrm{~h}$. A volume of $20 \mu \mathrm{l}(10 \% \mathrm{v} / \mathrm{v})$ containing $5 \mathrm{mg} / \mathrm{ml}$ of MTT solution was added followed by incubation over 30 minutes. After removing the medium, the cells were lysed, and the absorbance of lysates was determined at 595 nm using an EnVision 2104 multilabel reader (Perkin Elmer Inc., Waltham, USA). The percentage of viable cells was calculated in comparison to the untreated cells (control).

In addition, the bioactivity of NGF via the outgrowth of neurites from PC12 cells to neural phenotype upon exposure to NGF was investigated. The PC12 cell differentiation assay has been applied to test the activity of NGF earlier (Johnson et al. 2008). The cells grow neurites when exposed to NGF at concentration higher than approximately $10 \mathrm{ng} / \mathrm{mL}$ [32]. For this purpose, $5 \times 10^{4}$ cells per well were seeded in 24-well plates. NGF solution in PBS containing residues of poly ethylene glycol, HSA nanoparticles without NGF and PBS solution were used as controls. All reference samples containing NGF were studied with the following NGF at concentrations of 6.25 $\mathrm{ng} / \mathrm{ml}, 12.5 \mathrm{ng} / \mathrm{ml}, 25 \mathrm{ng} / \mathrm{ml}, 50 \mathrm{ng} / \mathrm{ml}, 100 \mathrm{ng} / \mathrm{ml}, 500 \mathrm{ng} / \mathrm{ml}, 1000 \mathrm{ng} / \mathrm{mL}$. Neurite outgrowth was examined and imaged by phase-contrast microscopy, and representative cells were photographed with a CCD camera (Zeiss, Oberkochen, Germany) after 3 days of exposure to the nanocarriers or pure growth factor. 


\subsection{Transport studies of theranostic nanoparticles using an in vitro BBB model}

A number of 10,000 bEND3 cells were seeded in a $100 \mu \mathrm{l}$ culture medium on 24 well $1 \mu \mathrm{m}$ ThinCerts which were precoated with $100 \mu \mathrm{l}$ FBS for $30 \mathrm{~min}$. In the lower compartment (basolateral), $1 \mathrm{ml}$ of culture medium, 10\% FBS (heat inactivated), Lglutamine and Na-pyruvate was added. As a control, half of the ThinCerts were not seeded with cells. ThinCerts with and without cells were incubated in the laminar flow bench at room temperature for one hour (to allow cells settling down) and subsequently for two hours at $37^{\circ} \mathrm{C}$. Afterwards, they were transferred to the cellZscope2 (nanoAnalytics, Münster, Germany). Two hundred microliters of medium were added to the ThinCerts to reach a final volume of $300 \mu \mathrm{l}$ in the upper compartment (apical). The transendothelial electrical resistance (TER) and capacitance were measured every hour for 5 days. After 5 days the medium was changed to DMEM with $2 \%$ FBS, $2 \mathrm{mM}$ L-glutamine, Na-pyruvate and $110 \mathrm{nM}$ hydrocortisone. Hydrocortisone was added to increase and to stabilize the TER value. The TER and capacitance values were measured every hour for 1 day. A volume of $17 \mu$ of the theranostic targeted nanoparticles, as well as the PEGylated reference formulation (without ApoE coating) were added to the cells (apical). The TER and capacitance values were recorded every 30 minutes for one day. A volume of $200 \mu \mathrm{l}$ of the nanoparticle samples (basolateral) were transferred to a black 96 well plate and measured in a plate reader using an excitation wavelength of $646 \mathrm{~nm}$ and an emission wavelength of $664 \mathrm{~nm}$ (ENSPIRE $\circledast$ Multimode plate reader, Perkin Elmer,Waltham, USA).

\subsection{Ethics}

All experiments were carried out in strict accordance with the guidelines for the European Community Council directive (2010/63/EU) for protection of vertebrate animals used for experimental and other scientific purposes. The surgeries were approved by Lund County Administrative Court under the auspices of the Swedish Department of Agriculture (M188-12). The study complies with the ARRIVE guidelines (Animal Research: Reporting In Vivo Experiments). 


\subsection{Animals and treatments}

Male Wistar rats obtained from Janvier were used for the experiments. Transient middle cerebral artery occlusion (tMCAO) model was induced for 2 hours followed by reperfusion. All animals were randomly divided into 3 groups; (i) vehicle treated, (ii) treated with U0126 and (iii) treated with NGF in combination with U0126. The MEK1/2 inhibitor U0126 was given at 0 and $24 \mathrm{~h}$ post reperfusion and NGF was given at day 3 post-stroke. Animals were randomly selected for treatment that was blinded to the operator.

\subsection{Transient middle cerebral artery occlusion model}

The experimental stroke model was performed by using an intraluminal filament technique [33] to induce transient middle cerebral artery occlusion in male Wistar rats. Anesthesia was induced by 3.5\% isoflurane in $\mathrm{N}_{2} \mathrm{O}: \mathrm{O}_{2}(70: 30)$ and then maintained by inhalation of $1.5-2 \%$ isoflurane in $\mathrm{N}_{2} \mathrm{O}: \mathrm{O}_{2}(70: 30)$ during the surgical procedure. The measurement of blood pressure, $\mathrm{pH}, \mathrm{pCO}_{2}, \mathrm{pO}_{2}$ and blood glucose was performed prior to the occlusion. To maintain body temperature during the surgical procedure a rectal temperature probe connected to a homoeothermic blanket was used. An incision was made in the midline of the neck, and the right common, internal and external carotid arteries were exposed. The right common and external carotid arteries were ligated permanently with sutures. A silicon rubber-coated monofilament (Doccol Corporation, Sharon, USA) was inserted into the right internal carotid artery and further advanced until the tip of the filament reached the entrance of the middle cerebral artery. The resulting occlusion was confirmed by Laser-Doppler flowmetry. Average LaserDoppler flow reductions of cerebral blood flow were $71 \pm 2 \%$. Following insertion of the filament the rats were sewed and regained consciousness. Two hours after occlusion, the rats were re-anesthetized and the filament removed to achieve reperfusion as verified by Laser-Doppler flowmetry. At the end of the surgery, the rats received a subcutaneous injection of $10 \mathrm{ml}$ of isotonic saline for hydration.

\subsection{Pre-clinical MR imaging at $9.4 T$ to evaluate infarct volume}

MRI monitoring was performed at day 14 post-stroke on a $9.4 \mathrm{~T}$ preclinical MRI horizontal bore scanner (Biospec Bruker,Billerica, USA) in conjunction with the 205/120 HD gradient coil unit. During the imaging procedure, the breathing rate and body temperature were monitored. The imaging was performed accordingly to 
previous described protocols [29]. The ${ }^{1} \mathrm{H} \mathrm{T}_{2}$-weighted images were acquired to measure the infarct volume. Images were analyzed using a self-written script in Matlab $^{\circledR}$ (Natick, Massachusetts, USA). The region of interest (ROI) corresponding to the whole ischemic lesion was manually delineated by an operator blinded to the treatment group and the extension of damage was reported as infarct volume $\left(\mathrm{mm}^{3}\right)$.

\subsection{Statistics}

All outcomes were expressed as the mean value \pm standard deviation (SD). The experiments (except the preformulation studies) were performed in triplicate at least (single experiments are indicated in the figure legend). The mean value and SD were analyzed by using Microsoft Excel (Microsoft, Redmond, USA). Particle characterization data was analyzed and illustrated using SigmaPlot 17.0 (Systat Software $\mathrm{GmbH}$, Germany). Statistical significance of various processes on particle size and in vivo experiments was analyzed using a one-way ANOVA test. Where indicated different statistical tests were used. 


\section{Results}

Theranostic NGF-loaded nanocarriers were manufactured by embedding USPIO and NGF into HSA core particles followed by surface modification with ApoE. The release of NGF was investigated with the help of the dispersion releaser technology [26]. The nanocarriers were evaluated for NGF bioactivity and cytotoxicity in PC12 cells and the transport through the BBB was studied in vitro using bEnd3 cells. The effect of a combination treatment of NGF with the small-molecular MEK inhibitor U0126 was studied in an animal model of stroke.

\subsection{Preparation of advanced NGF-loaded theranostic nanocarriers}

The diagnostic MRI-enabling maghemite $\left(\gamma-\mathrm{Fe}_{2} \mathrm{O}_{3}\right)$-based iron oxide particles were synthesized by two consecutive nanofabrication steps, the preparation of ultra-small basic Massart magnetite $\left(\mathrm{Fe}_{3} \mathrm{O}_{4}\right)$ nanoparticles followed by an optimized surface engineering method as described previously [23]. The nanoparticles were characterized for size by transmission electron microscopy (TEM) (6.61 \pm 2.04$)$ and by DLS $(60 \pm 2 \mathrm{~nm}, 0.136)$ as presented in Figure 1. It should be noted that, the hydrodynamic diameter measured by DLS is systemically larger than the one observed in TEM micrographs [34].
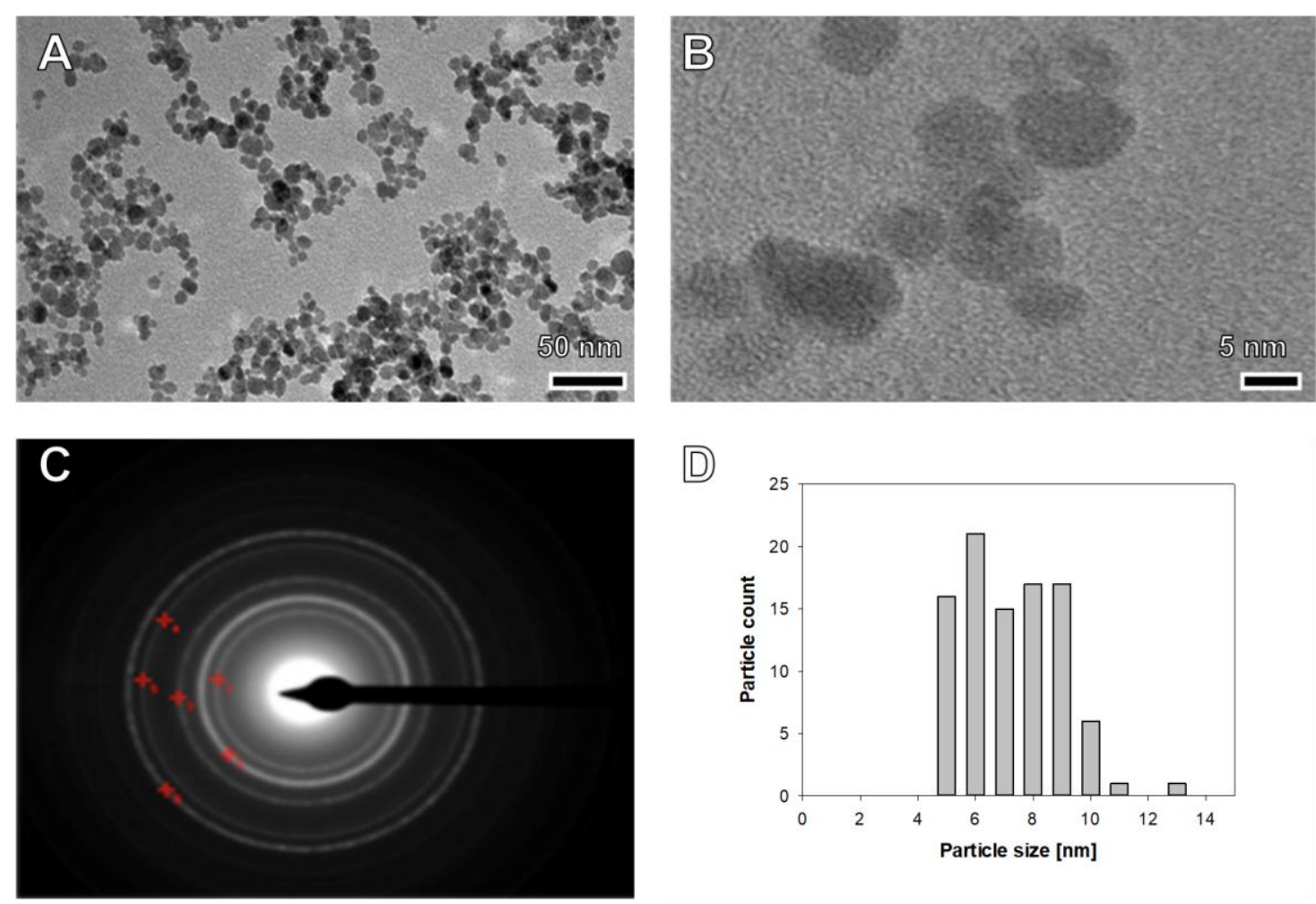

D)

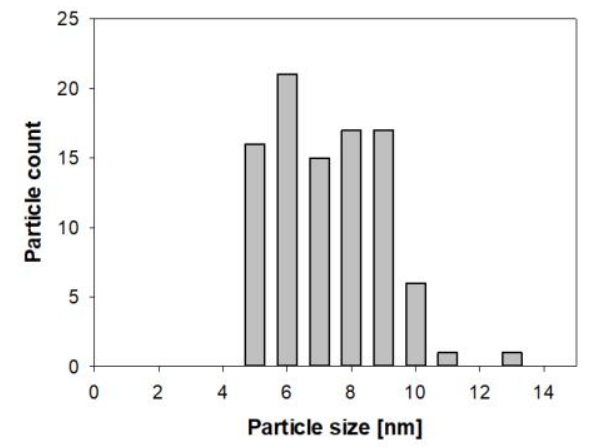

Figure 1: Standard $(A)$ and high-resolution (B) transmission electron micrograph, selected diffraction (SAED) pattern $(C)$ and size distribution histogram $(D)$ of ultra-small $6.61 \pm 2.04 \mathrm{~nm}$-sized USPIO. 
A zeta potential value of $+45.7 \pm 1.88 \mathrm{mV}$ together with a Ce/Fe ratio of 0.1000 was obtained. To encapsulate USPIO into albumin nanocarriers, the desolvation method was applied [35]. Presumably both, a positive surface charge and $\mathrm{Ce} 3 / 4^{+}$cations coordinative linkage led to a strong interaction with the negatively charged matrix material [22].

In previous designs, the HSA-CAN maghemite hybrid particles were optimized with an average diameter of $170 \pm 10 \mathrm{~nm}$ [21]. However, particle size differed significantly, when NGF was present. When adjusting an initial iron content of $1 \%(\mathrm{~m} / \mathrm{m})$ a particle size between $141 \pm 1 \mathrm{~nm}(\mathrm{pH} 6.5)$ and $207 \pm 1 \mathrm{~nm}(\mathrm{pH} 8.5)$ was obtained (see Figure 2 , left). Elevated $\mathrm{pH}$ values resulted in a considerable reduction of the particle yield, which was even more pronounced in presence of sodium chloride. Therefore, nanoparticle synthesis was conducted in absence in sodium chloride

Finally, theranostic nanocarriers were synthesized at a pH of 8.0. The HSA nanoparticles exhibited a hydrodynamic diameter of $152 \pm 6 \mathrm{~nm}$ (PDI $0.037 \pm 0.009$ ) and a particle yield of $75.3 \pm 4.9 \%$ yield $(\mathrm{m} / \mathrm{m})$. A zeta potential of $-47.7 \pm 1.4 \mathrm{mV}$ indicated high physical stability of the dispersion. In presence of NGF, a particle diameter of $191 \pm 2 \mathrm{~nm}$ (PDI $0.021 \pm 0.017$ ) with a particle yield of $71.5 \%(\mathrm{~m} / \mathrm{m})$ and a zeta potential of $-48.3 \mathrm{mV}$ was obtained.
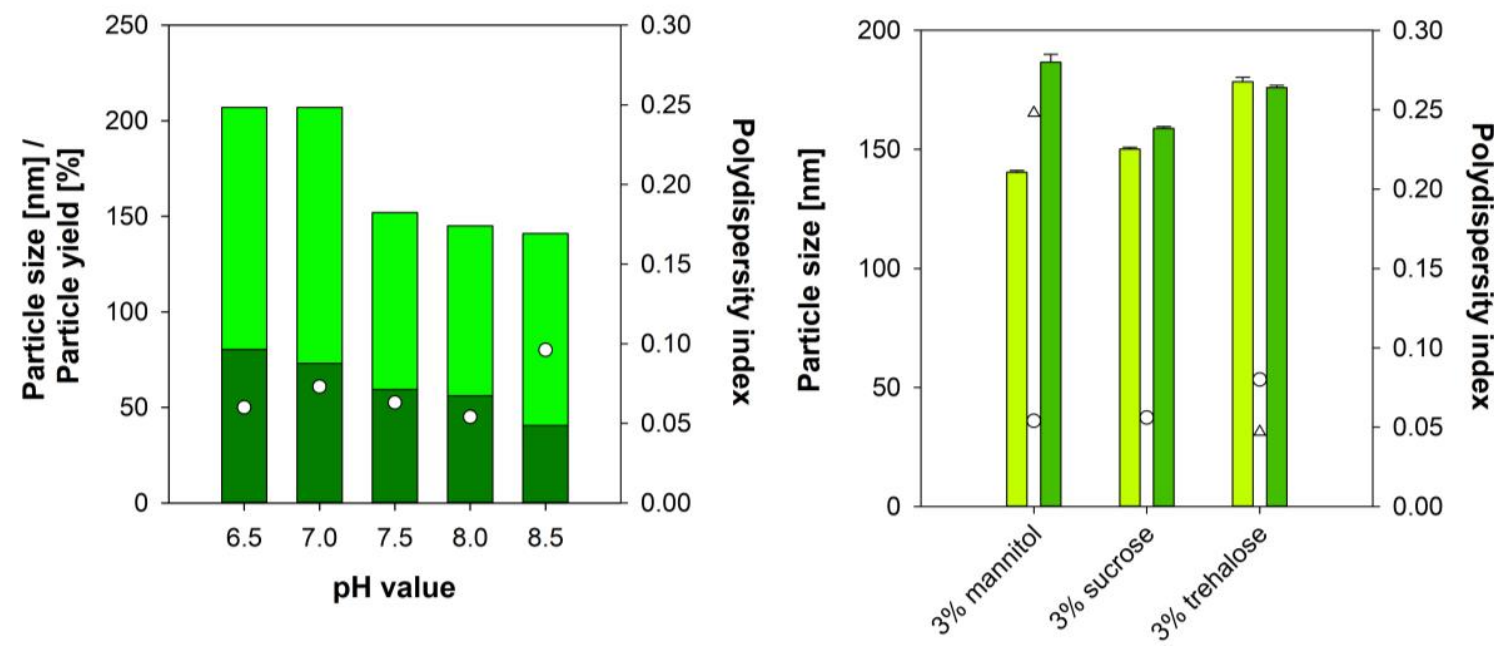

Figure 2: Particle size, particle yield, and polydispersity index of nanoparticles in the preformulation studies (left) synthesized at different $\mathrm{pH}$ values (particle size: light green bar, particle yield: dark green bar, $P D I$ : white dot) $(n=1)$. Particle size and polydispersity index of nanoparticles (right) before (particle size: light green bar, PDI: white dot) and after (particle size: dark green bar, PDI: white triangle) lyophilisation using mannitol, sucrose or trehalose as a lyoprotector ( $n=3$, mean $\pm S D)$. 
The conjugation with ApoE resulted in a slight but not significant (ANOVA) increase in particle size to $212 \pm 1 \mathrm{~nm}$ (PDI $0.075 \pm 0.022$ ).

Morphology of the targeted theranostic nanocarriers was explored by TEM. As expected, USPIO were embedded into the spherical cross-linked matrix system comprising HSA and NGF (see Figure 3). Finally, the magnetization of the theranostic nanocarriers, that contained $1.6 \%(\mathrm{w} / \mathrm{w})$ and $4.6 \%(\mathrm{w} / \mathrm{w})$ iron, was examined using a SQUID magnetometer. Converting the iron content to $100 \%$ of CAN-maghemite the resulting saturation magnetization values $(53.0 \mathrm{emu} / \mathrm{g}$ and $54.1 \mathrm{emu} / \mathrm{g})$ were comparable to those of CAN-maghemite $(59.5 \mathrm{emu} / \mathrm{g})$.
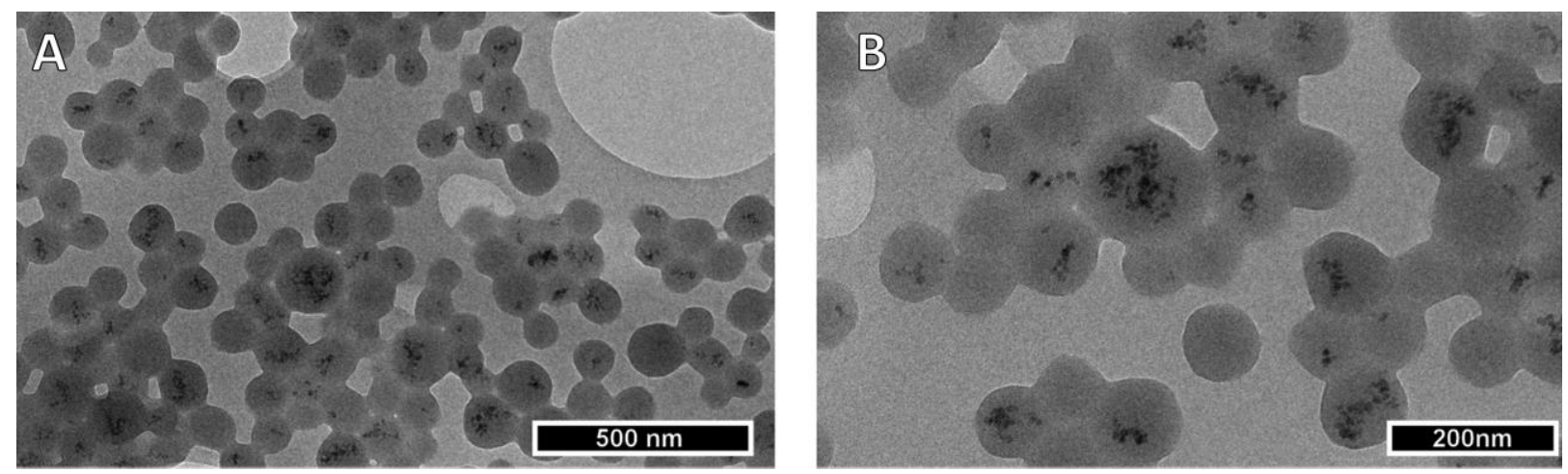

Figure 3: Transmission electron micrograph of targeted theranostic nanocarriers at 10000-fold (A) and 20,000-fold (B) magnification.

\subsection{Storage stability or nanosuspensions after freeze drying}

The initial formulation studies revealed a lyoprotector concentration of $3 \%$ as beneficial for the lyophilisation process. Therefore, the hybrid particles were freeze-dried in presence of $3 \%(\mathrm{w} / \mathrm{v})$ mannitol, sucrose and trehalose at a nanoparticle concentration of $10 \mathrm{mg} / \mathrm{ml}$ and tested for their physical stability after 6 months of storage at $4{ }^{\circ} \mathrm{C}$ and redispersion in MilliQ water. In the presence of sucrose and trehalose, the stability was maintained as indicated by particle size and PDI (see Figure 2, right). However, in the presence of mannitol, the particle size and PDI were considerably increased. Therefore, for further studies, all preparations were freeze-dried in the presence of sucrose.

3.3. In vitro characterization of theranostic nanocarrier formulations 
To exclude acute toxicity of the novel albumin-based targeted nanocomposites, the biocompatibility was tested. Cell viability was compared to a reference of untreated cells after an incubation time of 24,48 and $72 \mathrm{~h}$ (see Figure 4).

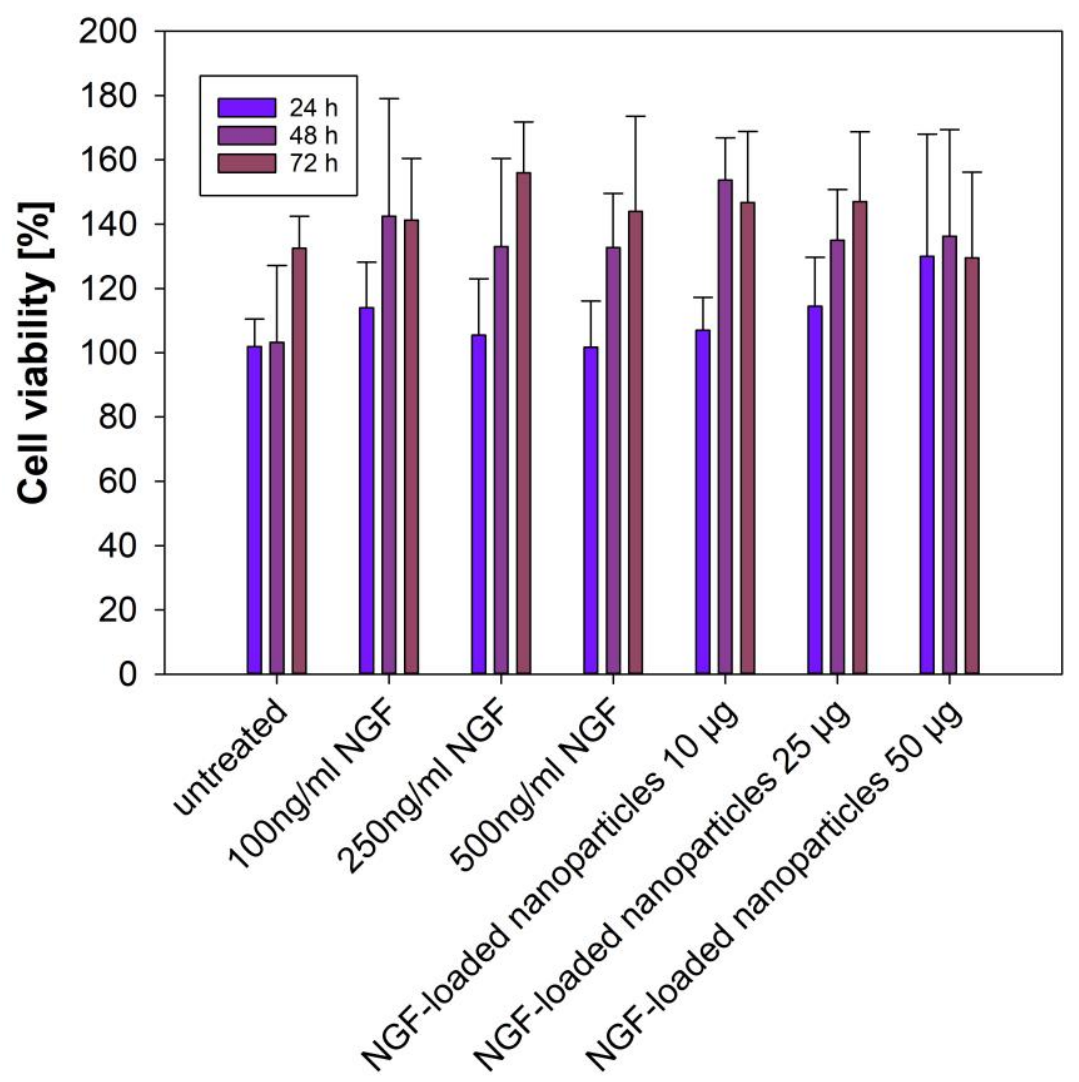

Figure 4: Cell viability of PC12 cells treated with $100 \mathrm{ng}, 250 \mathrm{ng}$ and $500 \mathrm{ng}$ of NGF as well as $10 \mu \mathrm{g}, 25 \mu \mathrm{g}$, and $50 \mu \mathrm{g}$ of NGF-loaded nanoparticles after 24,48 and $72 \mathrm{~h}$ of incubation. The viable cell mass was measured by the MTT assay. $(n=4$, mean $\pm S D)$.

This investigation was complemented by an evaluation of the bioactivity of NGF after encapsulation into the nanocarrier system. After several steps of processing including desolvation, chemical crosslinking, the surface modification, and lyophilisation, an influence on the bioactivity of the growth factor was assumed. The capacity of PC12 cells for neurite outgrowth has been used this way earlier [32].

Interestingly, the surface modification of NGF with polyethylene glycol (PEG) contributed to an enhanced stability of the protein [32] (see Figure 5D). The processing of NGF resulted in a substantial loss of biological activity. Neurite outgrowth was observed at more than an order of magnitude higher theoretical NGF concentration 
(500 $\mathrm{ng} / \mathrm{ml}$, see Figure $5 \mathrm{~F}$ ) in comparison to NGF solution or lyophilised and reconstituted NGF $(12.5 \mathrm{ng} / \mathrm{ml}$, see Figure $5 \mathrm{~B}, \mathrm{D})$.

To investigate the reasons behind the reduced activity, a drug release study was conducted using the dispersion releaser technology [26]. Compared to other methods, the dialysis-based setup allows a highly sensitive and reproducible measurement even in presence of serum proteins and other biorelevant media [27, 36]. The total drug release remained below $20 \%$ during the first 4 hours reaching a maximum value of $17.98 \pm 17.82 \%$. After 6 hours, proteinase $\mathrm{K}$ was added to the donor compartment in order to change the mechanism of release from diffusion to degradation [31]. As a result, release increased to $32.4 \pm 10.3 \%$.
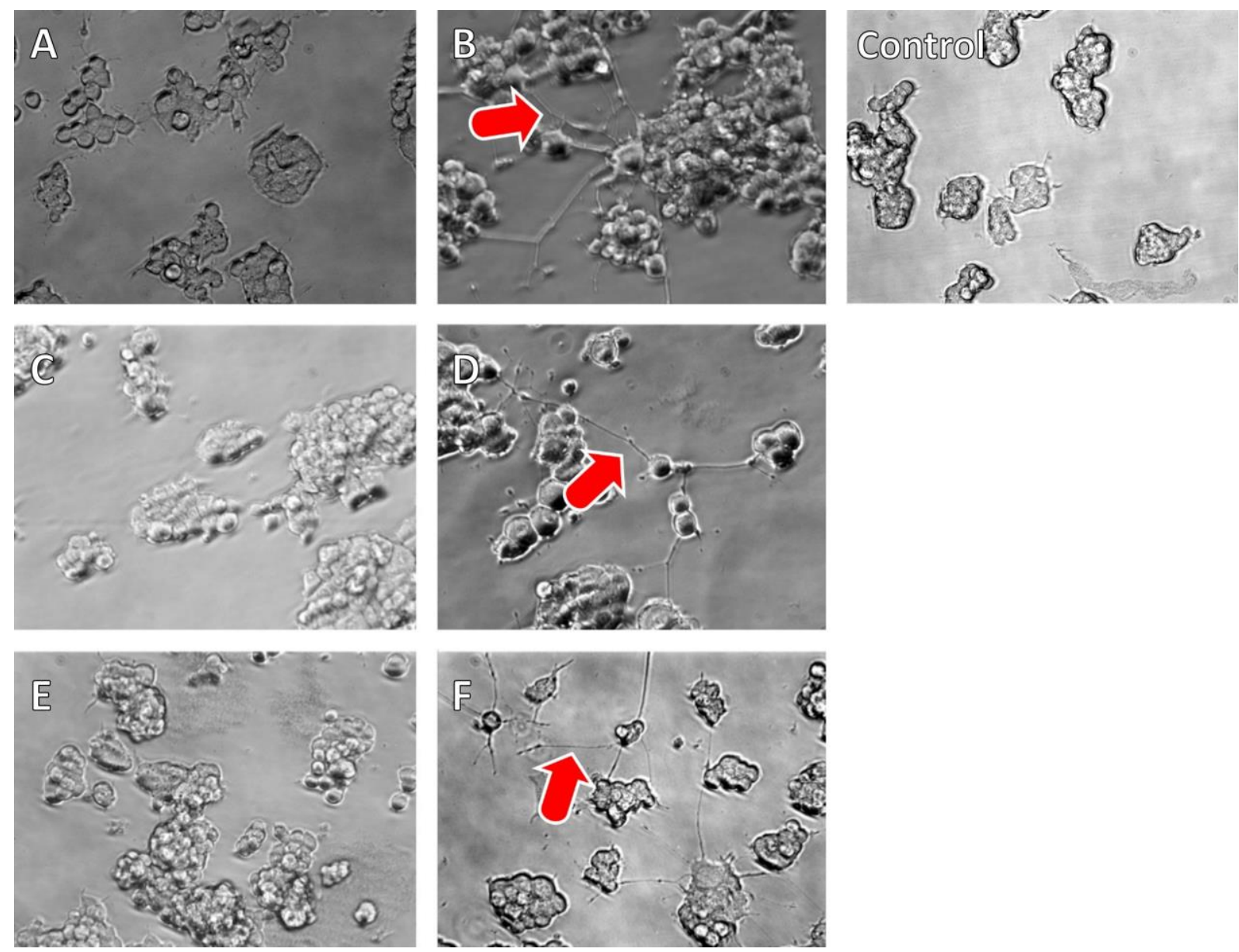

Figure 5: In vitro neurite outgrowth of rat embryonic pheochromocytoma cells (PC12) after incubation with NGFPEG solution (A: $6.25 \mathrm{ng} N \mathrm{NGF} /$ well, B: $12.5 \mathrm{ng}$ NGF/well), NGF-PEG lyophilisate solution (C: $6.25 \mathrm{ng}$ NGF/well, D: $12.5 \mathrm{ng}$ NGF/well), HSA-CAN maghemite-NGF-ApoE nanocomposite (E: $100 \mathrm{ng}$ NGF/well, F: 500 ng NGF/well) and blank HSA-CAN maghemite nanocomposite (Control: $50 \mu \mathrm{g}$ ). 
To complement these studies, the transport of nanocarrier formulations through an artificial BBB was investigated in vitro. For this purpose, a transwell assay system was applied using a confluent cell layer of the bEnd3 cell line. The TER value was monitored over the time of the experiment. A similar model has been used earlier to confirm the nanocarrier-mediated uptake of drugs into the brain [37]. After $48 \mathrm{~h}$ of incubation, the NGF concentration (measured as fluorescence intensity) was significantly higher in the basolateral compartment compared to the reference formulation without ApoE (see Figure 6A).
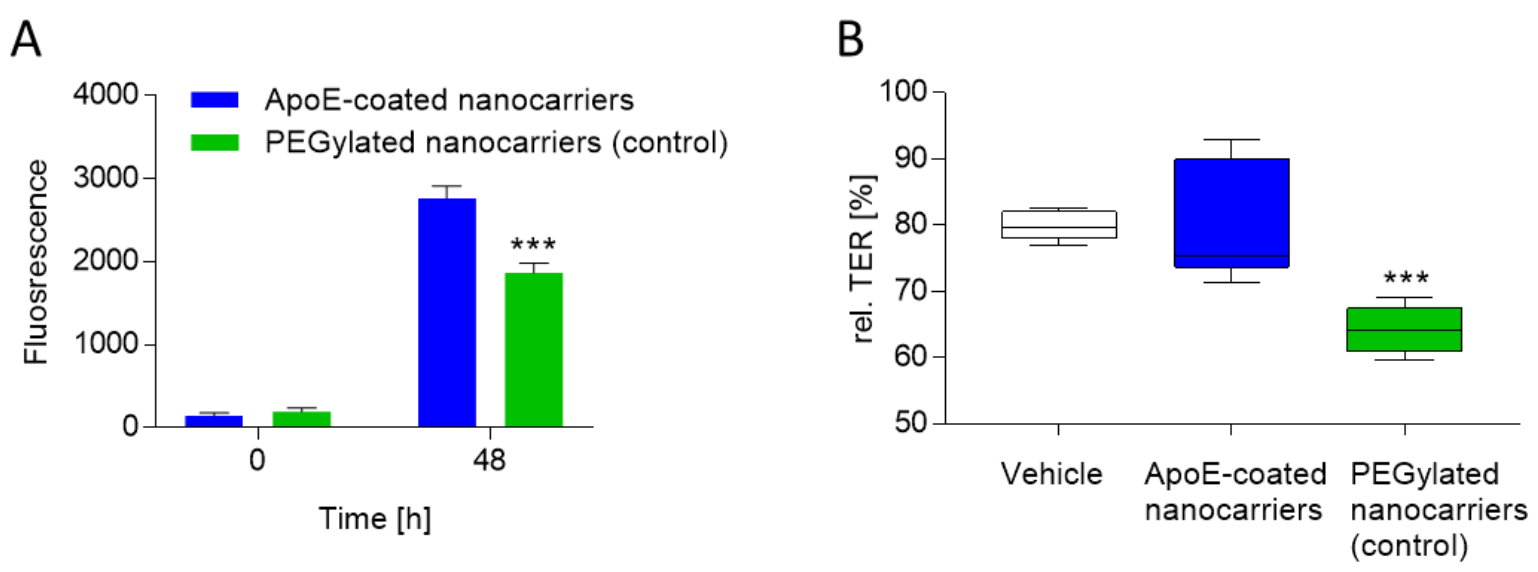

Figure 6: The penetration potential of nanocarriers was measured by detecting the fluorescence level in the basolateral compartment after $\mathrm{O}$ and $48 \mathrm{~h}$. ApoE-coated nanocarriers show an increased penetration in comparison to PEGylated nanocarriers (control). ${ }^{* * *} p<0.001$ indicates significant difference between ApoE-coated and PEGylated nanocarriers (t-test). B) To quantify changes of the TER, TER value $20 \mathrm{~h}$ after addition of the formulation (vehicle, ApoE-coated nanocarriers, PEGylated nanocarriers) was related to the TER value before the addition of the nanocarrier. The PEGylated nanocarriers reduce the TER value in comparison to the vehicle-treated control. ${ }^{* * *} p<0.001$ indicates significant difference between nanocarrier treated samples and vehicle treated samples (Bonferroni's multiple comparisons test).

The constant TER value indicated an intact barrier (see Figure 6B), however, there were some minor effects of the reference formulation on barrier integrity observed (see Figure 6B). This was not the case for the ApoE coated particles (see Figure 6B).

\subsection{Brain recovery after stroke}

Brain recovery after stroke was investigated inducing transient middle cerebral artery occlusion in male Wistar rats as described previously [33]. The brain infarct volume was determined from $\mathrm{T}_{2}$-weighted magnetic resonance images on day 14 post-stroke (see Figure 7A-C). A vehicle-control (see Figure 7A,D), the small molecule MEK inhibitor U0126 (see Figure 7D) and a combination of U0126 together with the NGFloaded drug delivery system were tested (see Figure 7C,D). 


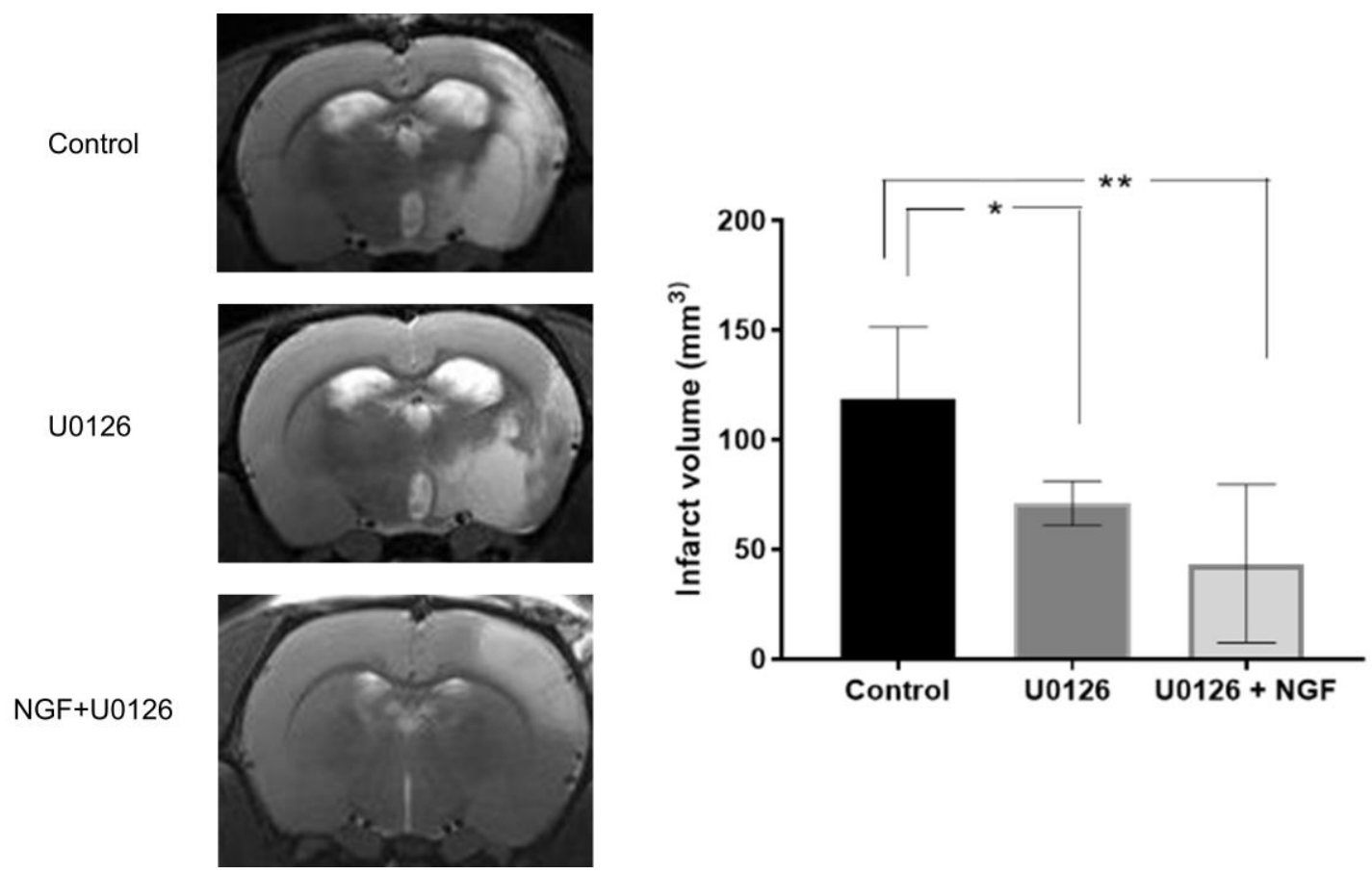

Figure 7: MR images of infarct site as well as the evaluation of infarct volume post-administration of vehicle control $(A, D)$, U126 $(B, D)$, and NGF-loaded nanocarriers in combination with U0126 $(C, D) 14$ days after stroke. $(n=9$, mean \pm SEM); * indicates significant differences between treatment and vehicle treated rats (ANOVA, $p<0.05)$. (Kruskal-Wallis test with Dunn's multiple comparisons analysis)

For the combination treatment using NGF-loaded theranostic nanocarriers and U0126, significant reduction of the infarct size was observed compared to the vehicle control (see Figure 7). U0126 alone also showed a significant reduction of the infarct size, however, there was no significant difference between the two treatments observed (see Figure 77).

\section{Discussion}

Today, stroke is a leading cause of death globally and is responsible for $9 \%$ of all deaths around the world [2]. A family of neurotrophins including NGF is involved in cerebral recovery [38] and was studied as a therapeutic option for brain injury [7]. Moreover, inhibition of MEK, a pathway which is activated following cerebral ischemia [39-42], in the acute phase of stroke, can improve the long-term neurological outcome $[29,43]$.

Unfortunately, NGF is not able to pass the BBB which effectively controls the cellular and molecular traffic between the brain and the periphery $[8,44]$. To bypass this barrier, previous studies utilized infusion into cerebral ventricles leading to a significant 
reduction of delayed neuronal death in rats [3]. In a clinical setting, there were two cases of infants reported where the intraventricular administration of NGF showed a beneficial effect on recovery of hypoxic-ischemic brain injuries and cerebral perfusion $[4,5]$.

Over the years, a broad array of methods has been developed to enhance access of drugs to the brain at therapeutically meaningful concentrations $[6,7]$. Nanocarrier delivery turned out to be an alternative to the conventional interventions. PBCA nanoparticles coated with polysorbate 80 were successfully used to deliver NGF to the brain after intravenous injection [13]. The adsorption of apolipoproteins followed by active transport through the BBB are the most likely mechanism [14]. Animals treated with the drug delivery system exhibited improved recognition and memory in the passive avoidance reflex (PAR) test [13].

In another study, transcytosis of targeted HSA nanoparticles through the brain endothelial cells was confirmed in vitro [15] and in vivo [12]. These particles were modified on their surface with ApoE [12, 15]. Even the short half-life of HSA nanoparticles in blood plasma [45] allowed the delivery system to access all six investigated brain regions [12].

In the present approach, nanocarriers for the treatment of stroke comprising a biodegradable matrix of HSA and NGF were modified on their surface with ApoE in order to deliver the growth factor to the brain. Further, USPIO were embedded into these nanoparticles to introduce diagnostic functionality to the delivery system. With an average diameter of $212 \pm 1 \mathrm{~nm}$ (PDI $0.075+/$-0.022), the nanoparticles were in a similar size range compared to earlier studies $(200-250 \mathrm{~nm})$ [12]. A monodisperse size distribution and optimal storage stability were achieved after freeze drying. As reported earlier, sucrose turned out to be an efficient lyoprotector for protein particles (see Figure 2, right) and was used to convert the liquid into a solid dispersion [30].

To track the in vivo biodistribution during later stages of preclinical and clinical translation, USPIO were co-encapsulated into the particle matrix. Initially, CAN maghemite particles were characterized for their particle size and size distribution (see section 3.1). As previously reported, the hydrodynamic diameter of USPIO (measured 
by DLS) was significantly larger compared to the one determined by electron microscopy [34].

Afterwards, successful encapsulation into the protein structure was visualized by TEM (see Figure 3). USPIO were homogeneously distributed and saturation magnetization values were comparable to the non-encapsulated suspension. The iron load was even higher compared to current literature [21]. Against this background, the particles might be suitable for MRI applications as required to explore biodistribution patterns [21].

As a next step, the toxicity and bioactivity of NGF-loaded nanocarriers was determined in vitro. After three days of incubation, the neurotrophin as well as the NGF-loaded nanocarriers induced neurite outgrowth in PC12 cells [32] suggesting that NGF remained active through the encapsulation procedure and was released from the carrier. Compared to NGF, the extent of this outgrowth was lowered for the nanoparticles (see Figure 5). The assay provides only semiquantitative information and more efforts will be made to study structure-activity relationships of the nanosystem. A delayed release of NGF from the carrier as well as a lowered activity following the processing and chemical modification of the protein can be assumed.

The in vitro release was studied using the dispersion releaser technology [26]. It remained below $20 \%$ during the first 4 hours $(17.98 \pm 17.82 \%)$. However, there was also a high variability observed between the three vessels which was in line with the rather complex release mechanism assumed for the carrier. With a molecular weight of approximately $24 \mathrm{kDa}$ the utilized model protein (trypsinogen) presumably had multiple interactions with the particle surface and pore structure of the nanocarrier which can lead to altered release patterns. After 6 hours, the release mechanism was shifted to a degradation-based release by adding proteinase $\mathrm{K}$ to the donor compartment. Degradation of HSA nanoparticles in presence of the enzyme has been studied previously [31]. A maximum release of $32.41 \pm 10.35 \%$ was achieved. The results confirm the outcome of the bioactivity assay showing a lowered activity but also explain the difficulties of applying in vitro findings to the in vivo situation. Further investigations of drug release as well as an optimization of the formulation parameters may be necessary. 
A number of studies suggested the delivery of NGF after transcytosis of the ApoEcoated nanoparticles through the BBB $[11,12,15]$. The LRP1 receptor was held responsible for this uptake [15]. In the present investigation, the transport of nanocarriers was investigated in vitro using a transwell assay system and bEnd 3 cells [37]. After $48 \mathrm{~h}$ the NGF concentration was significantly higher in the basolateral compartment for the ApoE-coated particles compared to the reference formulation (see Figure $6 \mathrm{~A}$ ). The TER value was slightly lowered for the control, indicating an influence of the reference on the barrier properties (see Figure $6 \mathrm{~B}$ ). Generally speaking, there are more advanced models of the BBB necessary, to detect the influence of slight formulation changes on drug transport [15]. For the ApoE-coated nanocarrier formulation a TER value of $79.81 \pm 8.58 \Omega . \mathrm{cm}^{2}$ was found. For comparison, in the in vivo setting, a TER value of $5900 \Omega . \mathrm{cm}^{2}$ has been detected. More recent cell-based models of the BBB achieve TER values of more than $500 \Omega . \mathrm{cm}^{2}$ [46]. However, using this model a transport mechanism was confirmed which has been reported for ApoEcoated particles in a number of studies $[15,47,48]$.

Finally, the efficacy of NGF-loaded nanocarriers was tested in combination with the small molecular MEK inhibitor U0126 in the transient middle cerebral artery occlusion in male Wistar rats [33]. The infarct size was significantly reduced compared to the vehicle control for the combination therapy as well as for U0126 alone.

Unfortunately, there was only a slight but not significant difference observed in the infarct size between the two therapies (combination and U0126 alone), presumably due to the small group size (see Figure 77). However, this trend was confirmed by the neurologic examination following the well-established 6-point and 28-point scoring systems [49-51] (Data not shown). This clearly suggests the effective delivery of NGF to the brain as well as a synergistic effect of the neuropeptide with the MEK inhibitor. The outcome of the neurologic examination and the histological evaluation will be published in a separate manuscript (Ansar et al. 2018, Manuscript in preparation). Further research will investigate the biodistribution of these theranostic nanocarriers using their superparamagnetic properties. Additionally, the therapeutic effect of NGFloaded nanocarriers will be tested alone to learn more about the mechanisms underlying this synergistic neuroprotection. 


\section{Conclusions}

The presence of the BBB poses an obstacle to drug delivery. Over the years, ways have been discovered to address specific transport mechanisms on the surface of the brain endothelial cells allowing therapeutics to enter the CNS. In the present study, we successfully encapsulated NGF and USPIO into an albumin matrix. These nanocomposites with a size of approximately $200 \mathrm{~nm}$ attained their superparamagnetic properties and exhibited low toxicity. After modification of the particle surface with $\mathrm{ApoE}$, the particles were able to cross an artificial BBB and remained bioactive in terms of the regulation of neurite outgrowth. In an animal model of stroke, a slight reduction of the infarct volume indicated a synergistic therapeutic effect of NGF and the smallmolecular MEK inhibitor U0126. The trend was also confirmed by an in-depth investigation of the neuroscore that will be published by Ansar and co-workers (manuscript in preparation). Further research will be necessary to study the biodistribution of this nanocarrier system as well as to understand the mechanisms underlying these observations.

\section{Acknowledgements}

The authors acknowledge Dr. Bernd Wolf for the SQUID measurements at the Institute of Physics (Goethe University, Frankfurt, Germany) as well as the Alexander von Humboldt Foundation (Ref. No.: 3.3-UNG/1161203 STP and 3.3-1161203-HUNHFST-E), the BIONANO_GINOP-2.3.2-15-2016-00017 project, the Swedish HeartLung Foundation and the LOEWE Research Centre for Translational Medicine and Pharmacology of the state of Hessen for financial support.

\section{References}

[1] L. Aloe, M.L. Rocco, B.O. Balzamino, A. Micera, Nerve Growth Factor: A Focus on Neuroscience and Therapy, Curr Neuropharmacol, 13 (2015) 294-303.

[2] G.A. Donnan, M. Fisher, M. Macleod, S.M. Davis, Stroke, The Lancet, 371 (2008) 1612-1623.

[3] T. Shigeno, T. Mima, K. Takakura, D. Graham, G. Kato, Y. Hashimoto, S. Furukawa, Amelioration of delayed neuronal death in the hippocampus by nerve growth factor, The Journal of Neuroscience, 11 (1991) 2914-2919.

[4] A. Chiaretti, O. Genovese, R. Riccardi, C. Di Rocco, D. Di Giuda, P. Mariotti, S. Pulitano, M. Piastra, G. Polidori, G.S. Colafati, L. Aloe, Intraventricular nerve growth 
factor infusion: a possible treatment for neurological deficits following hypoxic-ischemic brain injury in infants, Neurological research, 27 (2005) 741-746.

[5] C. Fantacci, D. Capozzi, P. Ferrara, A. Chiaretti, Neuroprotective role of nerve growth factor in hypoxic-ischemic brain injury, Brain sciences, 3 (2013) 1013-1022.

[6] L.T. Bonner, E.R. Peskind, Pharmacologic treatments of dementia, Med Clin North Am, 86 (2002) 657-674.

[7] P.M. Friden, L.R. Walus, P. Watson, S.R. Doctrow, J.W. Kozarich, C. Backman, H. Bergman, B. Hoffer, F. Bloom, A.C. Granholm, Blood-brain barrier penetration and in vivo activity of an NGF conjugate, Science (New York, N.Y.), 259 (1993) 373-377.

[8] D.J. Begley, ABC transporters and the blood-brain barrier, Curr Pharm Des, 10 (2004) 1295-1312.

[9] K. Ulbrich, T. Hekmatara, E. Herbert, J. Kreuter, Transferrin- and transferrinreceptor-antibody-modified nanoparticles enable drug delivery across the blood-brain barrier (BBB), Eur J Pharm Biopharm, 71 (2009) 251-256.

[10] K. Ulbrich, T. Knobloch, J. Kreuter, Targeting the insulin receptor: nanoparticles for drug delivery across the blood-brain barrier (BBB), J Drug Target, 19 (2011) 125132.

[11] A. Zensi, D. Begley, C. Pontikis, C. Legros, L. Mihoreanu, C. Buchel, J. Kreuter, Human serum albumin nanoparticles modified with apolipoprotein A-I cross the bloodbrain barrier and enter the rodent brain, J Drug Target, 18 (2010) 842-848.

[12] A. Zensi, D. Begley, C. Pontikis, C. Legros, L. Mihoreanu, S. Wagner, C. Buchel, $\mathrm{H}$. von Briesen, J. Kreuter, Albumin nanoparticles targeted with ApoE enter the CNS by transcytosis and are delivered to neurones, J Control Release, 137 (2009) 78-86.

[13] K.B. Kurakhmaeva, I.A. Djindjikhashvili, V.E. Petrov, V.U. Balabanyan, T.A. Voronina, S.S. Trofimov, J. Kreuter, S. Gelperina, D. Begley, R.N. Alyautdin, Brain targeting of nerve growth factor using poly(butyl cyanoacrylate) nanoparticles, J Drug Target, 17 (2009) 564-574.

[14] J. Kreuter, D. Shamenkov, V. Petrov, P. Ramge, K. Cychutek, C. Koch-Brandt, R. Alyautdin, Apolipoprotein-mediated transport of nanoparticle-bound drugs across the blood-brain barrier, J Drug Target, 10 (2002) 317-325.

[15] S. Wagner, A. Zensi, S.L. Wien, S.E. Tschickardt, W. Maier, T. Vogel, F. Worek, C.U. Pietrzik, J. Kreuter, H. von Briesen, Uptake mechanism of ApoE-modified nanoparticles on brain capillary endothelial cells as a blood-brain barrier model, PLoS One, 7 (2012) e32568.

[16] S. Gaca, S. Reichert, G. Multhoff, M. Wacker, S. Hehlgans, C. Botzler, M. Gehrmann, C. Rodel, J. Kreuter, F. Rodel, Targeting by cmHsp70.1-antibody coated and survivin miRNA plasmid loaded nanoparticles to radiosensitize glioblastoma cells, J Control Release, 172 (2013) 201-206.

[17] I.M. Steinhauser, K. Langer, K.M. Strebhardt, B. Spankuch, Effect of trastuzumabmodified antisense oligonucleotide-loaded human serum albumin nanoparticles prepared by heat denaturation, Biomaterials, 29 (2008) 4022-4028.

[18] M.G. Anhorn, H.C. Mahler, K. Langer, Freeze drying of human serum albumin (HSA) nanoparticles with different excipients, Int J Pharm, 363 (2008) 162-169.

[19] S. Dreis, F. Rothweiler, M. Michaelis, J. Cinatl, Jr., J. Kreuter, K. Langer, Preparation, characterisation and maintenance of drug efficacy of doxorubicin-loaded human serum albumin (HSA) nanoparticles, Int J Pharm, 341 (2007) 207-214.

[20] J.Y. Lee, K.H. Bae, J.S. Kim, Y.S. Nam, T.G. Park, Intracellular delivery of paclitaxel using oil-free, shell cross-linked HSA--multi-armed PEG nanocapsules, Biomaterials, 32 (2011) 8635-8644. 
[21] I. Rosenberger, C. Schmithals, J. Vandooren, S. Bianchessi, P. Milani, E. Locatelli, L.L. Israel, F. Hubner, M. Matteoli, J.P. Lellouche, M.C. Franchini, L. Passoni, E. Scanziani, G. Opdenakker, A. Piiper, J. Kreuter, Physico-chemical and toxicological characterization of iron-containing albumin nanoparticles as platforms for medical imaging, J Control Release, 194 (2014) 130-137.

[22] M.G. Wacker, M. Altinok, S. Urfels, J. Bauer, Nanoencapsulation of ultra-small superparamagnetic particles of iron oxide into human serum albumin nanoparticles, Beilstein J Nanotechnol, 5 (2014) 2259-2266.

[23] A.H. Haviv, J.M. Greneche, J.P. Lellouche, Aggregation control of hydrophilic maghemite (gamma-Fe2O3) nanoparticles by surface doping using cerium atoms, J Am Chem Soc, 132 (2010) 12519-12521.

[24] L.L. Israel, E. Lellouche, S. Ostrovsky, V. Yarmiayev, M. Bechor, S. Michaeli, J.P. Lellouche, Acute in vivo toxicity mitigation of PEl-coated maghemite nanoparticles using controlled oxidation and surface modifications toward siRNA delivery, ACS Appl Mater Interfaces, 7 (2015) 15240-15255.

[25] J.P. Lellouche, S. Michaeli, L.L. Israel, E. Lellouche, K. Buchman, Magnetic Inorganic Iron-Based Nanoparticles - Generalities and Use in Drug Delivery, in, 2014. [26] C. Janas, M.P. Mast, L. Kirsamer, C. Angioni, F. Gao, W. Mantele, J. Dressman, M.G. Wacker, The dispersion releaser technology is an effective method for testing drug release from nanosized drug carriers, Eur J Pharm Biopharm, 115 (2017) 73-83. [27] F. Jung, L. Nothnagel, F. Gao, M. Thurn, V. Vogel, M.G. Wacker, A comparison of two biorelevant in vitro drug release methods for nanotherapeutics based on advanced physiologically-based pharmacokinetic modelling, Eur J Pharm Biopharm, 127 (2018) 462-470.

[28] H. Ahnstedt, M. Mostajeran, F.W. Blixt, K. Warfvinge, S. Ansar, D.N. Krause, L. Edvinsson, U0126 attenuates cerebral vasoconstriction and improves long-term neurologic outcome after stroke in female rats, J Cereb Blood Flow Metab, 35 (2015) 454-460.

[29] M. Mostajeran, L. Edvinsson, K. Warfvinge, R. Singh, S. Ansar, Inhibition of mitogen-activated protein kinase $1 / 2$ in the acute phase of stroke improves long-term neurological outcome and promotes recovery processes in rats, Acta Physiol (Oxf), 219 (2017) 814-824.

[30] M. Wacker, K. Chen, A. Preuss, K. Possemeyer, B. Roeder, K. Langer, Photosensitizer loaded HSA nanoparticles. I: Preparation and photophysical properties, Int J Pharm, 393 (2010) 253-262.

[31] K. Langer, M.G. Anhorn, I. Steinhauser, S. Dreis, D. Celebi, N. Schrickel, S. Faust, V. Vogel, Human serum albumin (HSA) nanoparticles: reproducibility of preparation process and kinetics of enzymatic degradation, Int J Pharm, 347 (2008) 109-117.

[32] P.J. Johnson, S.L. Skornia, S.E. Stabenfeldt, R.K. Willits, Maintaining bioactivity of NGF for controlled release from PLGA using PEG, J Biomed Mater Res A, 86 (2008) 420-427.

[33] H. Memezawa, H. Minamisawa, M.L. Smith, B.K. Siesjo, Ischemic penumbra in a model of reversible middle cerebral artery occlusion in the rat, Exp Brain Res, 89 (1992) 67-78.

[34] J. Lim, S.P. Yeap, H.X. Che, S.C. Low, Characterization of magnetic nanoparticle by dynamic light scattering, Nanoscale Research Letters, 8 (2013) 381-381.

[35] C. Weber, J. Kreuter, K. Langer, Desolvation process and surface characteristics of HSA-nanoparticles, Int J Pharm, 196 (2000) 197-200.

[36] M. Villa Nova, C. Janas, M. Schmidt, T. Ulshoefer, S. Grafe, S. Schiffmann, N. de Bruin, A. Wiehe, V. Albrecht, M.J. Parnham, M. Luciano Bruschi, M.G. Wacker, 
Nanocarriers for photodynamic therapy-rational formulation design and medium-scale manufacture, Int J Pharm, 491 (2015) 250-260.

[37] S. Meister, I. Zlatev, J. Stab, D. Docter, S. Baches, R.H. Stauber, M. Deutsch, R. Schmidt, S. Ropele, M. Windisch, K. Langer, S. Wagner, H. von Briesen, S. Weggen, C.U. Pietrzik, Nanoparticulate flurbiprofen reduces amyloid-beta42 generation in an in vitro blood-brain barrier model, Alzheimers Res Ther, 5 (2013) 51.

[38] I.N. Solev, V.Y. Balabanyan, I.A. Volchek, O.S. Elizarova, S.A. Litvinova, T.L. Garibova, T.A. Voronina, Involvement of BDNF and NGF in the mechanism of neuroprotective effect of human recombinant erythropoietin nanoforms, Bulletin of experimental biology and medicine, 155 (2013) 242-244.

[39] J. Xing, J.M. Kornhauser, Z. Xia, E.A. Thiele, M.E. Greenberg, Nerve growth factor activates extracellular signal-regulated kinase and p38 mitogen-activated protein kinase pathways to stimulate CREB serine 133 phosphorylation, Molecular and cellular biology, 18 (1998) 1946-1955.

[40] S. Namura, K. lihara, S. Takami, I. Nagata, H. Kikuchi, K. Matsushita, M.A. Moskowitz, J.V. Bonventre, A. Alessandrini, Intravenous administration of MEK inhibitor U0126 affords brain protection against forebrain ischemia and focal cerebral ischemia, Proc Natl Acad Sci U S A, 98 (2001) 11569-11574.

[41] N. Sawe, G. Steinberg, H. Zhao, Dual roles of the MAPK/ERK1/2 cell signaling pathway after stroke, J Neurosci Res, 86 (2008) 1659-1669.

[42] G. Sun, N. Ye, D. Dai, Y. Chen, C. Li, Y. Sun, The Protective Role of the TOPK/PBK Pathway in Myocardial Ischemia/Reperfusion and $\mathrm{H}(2) \mathrm{O}(2)$-Induced Injury in H9C2 Cardiomyocytes, Int J Mol Sci, 17 (2016) 267.

[43] L.I. Edvinsson, G.K. Povlsen, Vascular plasticity in cerebrovascular disorders, J Cereb Blood Flow Metab, 31 (2011) 1554-1571.

[44] A. Nyul-Toth, M. Suciu, J. Molnar, C. Fazakas, J. Hasko, H. Herman, A.E. Farkas, J. Kaszaki, A. Hermenean, I. Wilhelm, I.A. Krizbai, Differences in the molecular structure of the blood-brain barrier in the cerebral cortex and white matter: an in silico, in vitro, and ex vivo study, American journal of physiology. Heart and circulatory physiology, 310 (2016) H1702-1714.

[45] E. Fahrlander, S. Schelhaas, A.H. Jacobs, K. Langer, PEGylated human serum albumin (HSA) nanoparticles: preparation, characterization and quantification of the PEGylation extent, Nanotechnology, 26 (2015) 145103.

[46] B. Srinivasan, A.R. Kolli, M.B. Esch, H.E. Abaci, M.L. Shuler, J.J. Hickman, TEER measurement techniques for in vitro barrier model systems, Journal of laboratory automation, 20 (2015) 107-126.

[47] M. Dadparvar, S. Wagner, S. Wien, J. Kufleitner, F. Worek, H. von Briesen, J. Kreuter, HI 6 human serum albumin nanoparticles--development and transport over an in vitro blood-brain barrier model, Toxicol Lett, 206 (2011) 60-66.

[48] S. Wagner, J. Kufleitner, A. Zensi, M. Dadparvar, S. Wien, J. Bungert, T. Vogel, F. Worek, J. Kreuter, H. von Briesen, Nanoparticulate transport of oximes over an in vitro blood-brain barrier model, PLoS One, 5 (2010) e14213.

[49] T. Engelhorn, S. Goerike, A. Doerfler, C. Okorn, M. Forsting, G. Heusch, R. Schulz, The angiotensin II type 1-receptor blocker candesartan increases cerebral blood flow, reduces infarct size, and improves neurologic outcome after transient cerebral ischemia in rats, J Cereb Blood Flow Metab, 24 (2004) 467-474.

[50] A. Encarnacion, N. Horie, H. Keren-Gill, T.M. Bliss, G.K. Steinberg, M. Shamloo, Long-term behavioral assessment of function in an experimental model for ischemic stroke, Journal of neuroscience methods, 196 (2011) 247-257. 


\section{First revision}

[51] J.B. Bederson, L.H. Pitts, M. Tsuji, M.C. Nishimura, R.L. Davis, H. Bartkowski, Rat middle cerebral artery occlusion: evaluation of the model and development of a neurologic examination, Stroke, 17 (1986) 472-476. 